\title{
MicroRNA variants and HLA-miRNA interactions are novel rheumatoid arthritis susceptibility factors
}

Shicheng Guo"\#, Yehua Jinn ${ }^{2,3 \#}$, Jieru Zhou" ${ }^{4 \#}$, Qi Zhu ${ }^{3,5 \#}$,Ting Jiang ${ }^{3}$, Yanqin Bian ${ }^{3,5}$, Runrun Zhang ${ }^{2,3}$, Cen Chang $^{2,3}$, Lingxia Xu ${ }^{2,3}$, Jie Shen ${ }^{3,5}$, Xinchun Zheng ${ }^{3}$, Yi Shen ${ }^{3}$, Yingying Qin ${ }^{3}$, Jihong Chen $^{3}$, Xiaorong Tang ${ }^{3}$, Peng Cheng ${ }^{3}$, Qin Ding ${ }^{3}$, Yuanyuan Zhang ${ }^{3}$, Jia Liu' ${ }^{3}$, Qingqing Cheng, ${ }^{3}$, Mengru Guo ${ }^{3}$, Zhaoyi Liu $^{3}$, Weifang $\mathrm{Qiu}^{3}$, Yi Qian ${ }^{3}$, Yang Sun ${ }^{3,5}$, Yu Shen ${ }^{3,5}$, Hong Nie $^{6}$, Steven J Schrodi ${ }^{1 \star}$, Dongyi He ${ }^{3,5 *}$

1. Department of Medical Genetics, School of Medicine and Public Health, University of Wisconsin-Madison, Madison, WI, USA. 2, Shanghai University of Traditional Chinese Medicine, Shanghai, China. 3, Department of Rheumatology, Shanghai Guanghua Hospital of Integrated Traditional Chinese and Western Medicine, Shanghai, China. 4, Department of Health Management, Shanghai East Hospital, Tongji University School of Medicine,Shanghai,China.5, Arthritis Institute of Integrated Traditional Chinese and Western Medicine,Shanghai Academy of Traditional Chinese Medicine, Shanghai University of Traditional Chinese Medicine. 6, Shanghai Institute of Immunology, Shanghai Jiao Tong University School of Medicine, Shanghai, China

\#These authors contributed equally to this work

${ }^{*}$ Correspondence:

Steven J Schrodi, Ph.D.

Department of Medical Genetics

School of Medicine and Public Health

University of Wisconsin-Madison, Madison

Tel: 608-263-3068

Email: Schrodi@wisc.edu

Dongyi He, M.D., Ph.D.

Department of Rheumatology

ShangHai Guanghua Hospital of Integrated Traditional Chinese and Western Medicine, Shanghai, 200052, China

Tel : 158-0030-0800

Email: hedongyi1967@shutcm.edu.cn

Number of Words : 2938 


\section{Abstract}

Objective: Althogh Genome-wide association studies have identified $>100$ variants for rheumatoid arthritis $(\mathrm{RA})$, the reported genetic variants only explain $<40 \%$ of RA heritability. We conducted a systemic association study between common East-Asian miRNA SNPs with RA in a large Han Chinese cohort to explain missing heritability and identify miRNA epistatic interactions.

Methods: 4 HLA SNPs (HLA-DRB1, HLA-DRB9, HLA-DQB1 and TNFAIP3) and 225 common SNPs located in miRNA which might influence the miRNA target binding or pre-miRNA stability were genotyped in 1,607 rheumatoid arthritis and 1,580 matched normal individuals. A meta-analysis with previous GWAS studies ( 4,873 RA cases and 17,642 controls ) was performed to discovery another novel miRNA RA-associated SNPs.

Results: 2 novel SNPs including rs1414273 (miR-548ac, OR=0.84, P=8.26x10-4) and rs2620381 (miR-627, $\mathrm{OR}=0.77, \mathrm{P}=2.55 \times 10-3)$ conferred significant association with RA.Individuals carried 8 risk alleles showed 15.38 (95\%Cl: 4.69-50.49, $\mathrm{P}<1.0 \times 10-6$ ) times more risk to be affected by RA. In addition, rs5997893 (miR3928) showed significant epistasis effect with rs4947332 (HLA-DRB1, OR=4.23, P=0.04) and rs2967897 (miR5695) with rs7752903 (TNFAIP3, OR=4.43, $P=0.03$ ). Finally, we demonstrated targets of the significant miRNAs showed enrichment in immune related genes $(P=2.0 \times 10-5)$ and FDA approved drug target genes $(P=0.014)$.

Conclusions: 6 novel miRNA SNPs including rs1414273 (miR-548ac, $\mathrm{P}=8.26 \times 10-4)$, rs2620381 (miR-627, $\mathrm{P}=2.55 \times 10-3)$, rs4285314 (miR-3135b, $\mathrm{P}=1.10 \times 10-13$ ), rs28477407 (miR-4308, $\mathrm{P}=3.44 \times 10-5$ ), rs5997893 (miR-3928, $P=5.9 \times 10-3$ ) and $r 545596840$ (miR-4482, $P=6.6 \times 10-3$ ) were confirmed to be significantly associated with RA in a Chinese population. Our study suggests that miRNAs might be interesting targets to accelerate the understanding of the pathogenesis and drug development for rheumatoid arthritis.

Keywords: Rheumatoid Arthritis, miRNA, SNP, susceptibility, predispose

\section{Introduction}

Rheumatoid arthritis (RA) is a chronic inflammatory disorder caused by the interaction between multiple factors including genetics, epigenetics and the environment. ${ }^{1-3}$ Twin studies estimate that an RA heritability of $\sim 60 \%$. ${ }^{45}$ In the past 15 years, GWAS have identified several hundred RA-associated variants. However, the reported genetic variants only explain $<40 \%$ of RA heritability. ${ }^{5-8}$ Furthermore, studies within non-European-derived samples will aid our understanding of RA molecular pathophysiology as different variants and, potentially, different disrupted pathways may be present in those sample sets. Additionally, studying SNPs in specific functional classes of genes or motifs may reveal specific pathogenic mechanisms not previously discovered through GWAS. ${ }^{9}$ GWAS results indicate that $90 \%$ of disease-associated variants are located in non-coding regions, indicating regulatory elements may play important roles in complex disease etiology, including $R A .{ }^{10}$ Across regulatory elements, microRNAs are important targets to interrogate as miRNA SNPs may modify expression at numerous genes. Moreover, miRNAs play pivotal roles in both innate and adaptive immunity, ${ }^{11}{ }^{12}$ sex-specific effects, ${ }^{13}$ and disease onset. ${ }^{1415}$

Recently, several preliminary RA-association studies focused on miRNAs have been conducted in Asian, ${ }^{16-19}$ European $^{20}$ and African ${ }^{21}$ samples. However, these studies have very limited sample sizes and only included a subset of miRNAs without genotyping all miRNA common SNPs. Additionally, the previous RA GWAS in Han Chinese was conducted in $2014^{22}$ using an array with poor coverage of miRNA SNPs. Therefore, we conducted an exhaustive study of functional miRNA SNPs in RA. miRbase annotates 1,920 primary miRNA and 2,883 mature miRNAs. From dbSNP153, there are $>45,705$ SNPs located in primary miRNAs and 17,570 in mature miRNA; ${ }^{23}$ however, only 733 of these SNPs segregate alleles at moderate-high frequency (i.e. are common SNPs with MAF>1\%) within primary miRNAs regions. Within specific populations, this number is expected to be substantially reduced to 200 SNPs, providing an opportunity to use a multiplex genotyping assay in our RA samples. Hence, we conducted a systemic association study between common East-Asian 
miRNA SNPs with RA in a large Han Chinese cohort to identify novel miRNA SNPs and miRNA epistatic interactions.

\section{Methods}

\section{Precision Medicine Research Cohort in Shanghai Guanghua Hospital}

Guanghua Hospital Precision Medicine Research Cohort (PMRC) is a hospital-based longitudinal cohort to investigate risk factors, genetic susceptibility, pharmacogenetics for rheumatology diseases such as RA, osteoarthritis and ankylosing spondylitis. Healthy individuals are derived from those with an annual physical exam without rheumatological disease. Currently, PMRC has enrolled $>30,000$ disease patients and 10,000 healthy individuals as controls. We randomly selected 3,223 individuals including 1,625 seropositive (RF+ and anti-CCP+) RA and 1,598 controls from the PMRC. Written consent was collected prior to enrollment. Non-Han Chinese individuals were excluded from the study to avoid confounding by population stratification. All cases fulfilled the 2010 European League against Rheumatism-American College of Rheumatology criteria or 1987 American College of Rheumatology revised criteria for RA. All healthy controls were required do not have personal or family history of ankylosing spondylitis, rheumatoid arthritis, osteoarthritis, type 1 and 2 diabetes, chronic infection or common cancers. We did not find significant case/control difference between gender, age, smoking, BMI after correction for multiple testing. This study was reviewed and approved by the Institutional Review Board of Guanghua Hospital (No: IRB12018-K-12) and all methods were performed in accordance with the relevant guidelines and regulations. The demographic and clinical characteristics of the whole samples are presented in Table S1.

\section{DNA extraction, SNP selection and Genotyping}

Genomic DNA was extracted from peripheral blood using the CB-kit (CoWin Biosciences, CWBIO, China) according following manufacturer's instructions. Allelic discrimination was automated using the manufacturer's software which has been widely-described in previous studies. ${ }^{24}$ Internal positive and negative control samples were used and a test of Hardy-Weinberg equilibrium was employed to assess the genotyping quality (SNPS exceeding HWE p-value cutoff were excluded from RA association testing). Cases and controls were required to have a family history of at least three generations of residency in Shanghai or neighboring regions. The SNaPshot Multiplex System (Applied Biosystem,USA) was used for the genotyping which has been widely used in our previous studies. ${ }^{2526}$

To select SNPs for subsequent association testing, human microRNAs were downloaded from miRBase methjods (Release 22.1). Mapping of SNPs within dbSNP (dbSNP153, 08/08/2019) was performed for human microRNA genomic regions, resulting in 40,602 SNPs. To select common SNPs (MAF>0.01), we collected the allele frequency for all the SNPs from Gnomad, ${ }^{27}$ Asian $100 \mathrm{~K}^{28}$ and the 1000 Genomes dataset. ${ }^{10}$ The minor allele frequency was required to be higher than 0.01 in all datasets. [t] ${ }^{29}$ Only biallelic SNPs were included in the analysis, while tri-allelic SNPs were excluded from this study. Following filtering, 243 SNPs were obtained. In order to decrease the genotyping cost, one SNP from pairs of SNPs with high $L D\left(R^{2}>0.8\right)$ were removed and only one of them was included in the genotyping assay. A total of 233 SNPs were genotyped. GRCH37/hg19 was used to determine genomic positions and $R^{2}>0.6$ as the cut-off to selected imputed SNPs of high quality and located in miRNA regions. The GAsP reference panel showed higher imputation accuracy (93\%-95\%) compared with 1000 Genome Asian panel (<90\%). ${ }^{30}$ Four SNPs within known GWAS associations with RA were included: rs9268839 (HLA-DRB9), ${ }^{31} 32$ rs9275376 (HLA-DQB1), ${ }^{33-36}$ rs4947332 (tag-SNP for $\left.H L A-D R B 1^{*} 04: 07\right)$ and rs7752903 (TNFAIP3). ${ }^{31}{ }^{32}$ In addition, a panel of five ancestry informative markers (negative control) were selected to estimate the potential effects of population stratification: rs 174583 (11q12.2), rs11745587 (5q31.1), rs521188 (1p31.3) and rs7740161 (6q23.2). These SNPs were derived from a previous Han Chinese population study. ${ }^{37}$ These SNPs are informative for interrogating population structure between South and North Han Chinese since the Shanghai region is a mixed population from different regions in China (Figure 1A).

\section{Quality Control and Power analysis}


For genotyping QC, 2.5\% samples (40 RA and 32 normal) were randomly selected for repeat genotyping. The concordance between initial and replicate genotyping was $99.98 \%$. SNP imputation was accomplished through the Michigan Imputation Server (https://imputationserver.sph.umich.edu/index.html) with East Asian samples from the Genome Asian Pilot (GAsP). Missing ratio between case and control differential test and HWE in control samples were evaluated. SNPs were removed when $\mathrm{P}<0.01$.

In the power analysis, given the prevalence of RA $(p=0.01)$, case $(n=1,607)$ and control $(n=1,580)$ size, risk allele frequency $(f=0.1)$ and genetic effect size $(O R=1.5)$, the power for multiplicative model, additive model, dominant models were $0.856,0.823$ and 0.742 , respectively using a significance level of alpha $=5 \times 10^{-03}$. In addition, with our design and the above effect size, we calculate that SNPs with MAF of 0.025 can attain a statistical power of $80 \%$ with nominal $p<0.05$. Therefore, our study could provide reliable genetic association results for the majority of the alleles we have included given that the MAF of 201 (86.3\%) SNPs are $>10 \%$ and MAF of $96 \%$ included SNPs exceed $2.5 \%$.

\section{Bioinformatics analysis of miRNA regulatory networks and enrichment analysis}

To understand regulatory networks driven by miRNAs and the overlap with RA GWAS genes and immune system genes, a miRNA regulatory network analysis was performed with miRDB. ${ }^{38}$ Genes predicted to be regulated by significant miRNAs were calculated among 123 previously-identified RA GWAS genes, 4,723 immune system genes collected by InnateDB database ${ }^{39}$ and 373 FDA-targeted pharmacogenes. A target sore threshold was set to 95 in miRDB to identify reliable gene targets for the miRNAs. Cytoscape was employed to construct and visualize the miRNA-mRNA network. We calculated a hypergeometric test of the enrichment in miRNA targets within RA GWAS significant genes, immune-related genes, and FDA approved drug targets. A random sampling technique was applied to assess the null distribution of $p$-values $(n=50,000$ iterations). 37,875 total genes in the genome (GENCODE v32) were assumed for the calculations. Enrichment was measured with fold-change (FC) and p-values were calculated by summing the frequency of more extreme values in the null distribution of $p$-values.

\section{Association Analysis, Power Calculations, Epistasis Analysis and Cumulative Risk Analysis}

RA Association for each SNP were calculated and OR estimated $(95 \% \mathrm{Cl})$ using Chi-square test, Fisher's Exact test $(\mathrm{N}<5)$, Cochran-Armitage Trend test and Bayesian logistic regression $(\mathrm{BLR})^{40}$ adjusted for gender,

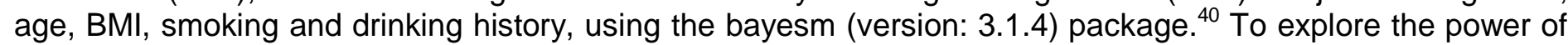
the study, a Monte Carlo simulation was performed under different models including dominant, recessive and additive models, additive models, using a Chi-square test or Bayesian logistic regression model. SNP-SNP interactions were analyzed by using traditional point-wise interaction analysis based on SNPassoc, ${ }^{41}$ logistic test, ${ }^{42}$ "fast-epistasis" in Plink. ${ }^{43}$ We applied $Y \sim b 0+b 1^{\star} A+b 2^{\star} B+b 3^{\star} A B+e$ to detect epistasis effect between SNP A and SNP B when b3 not equal 0 (null hypothesis: b3 equal 0). A $P<0.001$ was considered significant. An RA risk prediction model was fitted with the 6 most significant SNPs (rs1414273, rs4947332, rs9268839, rs9275376, rs7752903 and rs2620381) by both Chi-square and logistic regression test to adjust by above-mentioned covariates. Q-value received after multiple test correction was conducted through a False Discover Rate (FDR) implemented in $\mathrm{R}$ with the function p.adjust). ${ }^{44}$ SNP derived miRNA target gain or loss were imputed by miRNA-SNP database ${ }^{45}$ with the default setting. All the statistical analyses were performed with $\mathrm{R}$ (version: 3.6.1).

\section{Results}

\section{SNP Selection, Genotyping and Population Structure}

Overall, the genotyping generated high quality data with $94 \%$ of the SNPs showing high genotyping quality. Seven of the SNPs were removed from being promoted to the RA association evaluation due to a genotyping ratio less than $99.0 \%$. The missing ratio between case and control was calculated. Hardy-Weinberg equilibrium in control samples was evaluated and another 3 SNPs were removed when $P<0.01$. Finally, 223 SNPs in 1,607 rheumatoid arthritis and 1,580 normal individuals were used in the association analysis (genotyping rate=98.88\%). PCA analysis based on these SNPs showed our samples clustered with the East Asian population (Figure 1B). Furthermore, we did not identify significant age, BMI, drinking and smoking history 
differences between cases and controls ( $P>0.01$, Table S1). However, as a conservative measure, association testing of all the variants included age, gender, BMI, drinking and smoking as covariates in the Bayesian logistic regression (BLR)-based tests.

\section{Novel RA-associated SNPs located in miRNA-548 and miRNA-627}

Applying the Bayesian logistic regression model adjusted for covariates, we identified 6 significant SNPs located in HLA-DRB9 (rs9268839, $\left.\mathrm{P}=3.95 \times 10^{-27}\right), \quad H L A-D R B 1$ (rs4947332, $\left.\mathrm{P}=2.78 \times 10^{-4}\right), \quad H L A-D Q B 1$

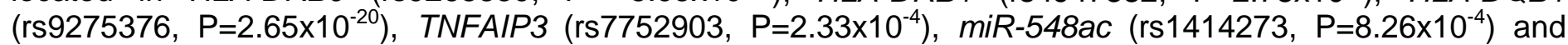
miR-627 (rs2620381, $\mathrm{P}=2.55 \times 10^{-3}$ ) and 15 additional SNPs with $\mathrm{P}<0.05$ (Table 1). As expected, all the SNPs located in HLA-DRB1, HLA-DRB9, HLA-DQB1 and TNFAIP3 were significantly associated with RA status, which is consistent with previous GWAS studies. We found all the population markers including rs 174583 (FDR=0.88), rs11745587 (FDR=0.98), rs521188 (FDR=0.99) and rs7740161 (FDR=0.48) were not significant in the association test between RA and control. Additionally, two miRNA SNPs located in miR-548ac (FDR=0.01) and miR-627 (FDR=0.045) were significantly associated with RA after FDR adjustment for multiple testing $(P<0.05$, FDR). We also conducted mode of inheritance-based association analyses (Table 2) and Cochran-Armitage Trend test-based association analyses (Table S2). We found a dominant model of two miRNA SNPs (rs1414273 and rs2620381) showed highly significant association between the alleles and RA status. To combine previous Han Chinese association data with this study, we also implemented a metaanalysis in which 4,873 RA cases and 17,642 normal individuals from the East Asian Population (EAP) were included. The meta-analysis resulted in an additional 4 significant SNPs (Table 3, Figure 2) including rs4285314 (miR-3135b, FDR $\left.=1.10 \times 10^{-13}\right)$, rs28477407 (miR-4308, FDR $\left.=3.44 \times 10^{-5}\right)$, rs5997893 (miR-3928, $\left.\mathrm{FDR}=5.9 \times 10^{-3}\right)$ and $\mathrm{rs} 45596840\left(\mathrm{miR}-4482\right.$, FDR $\left.=6.6 \times 10^{-3}\right)$. Finally, we also evaluated whether rs 1414273 (miR-548ac) and rs2620381 (miR-627) are significant RA susceptibility variants independent of HLA variants. We found that the association at $r s 1414273\left(\mathrm{OR}=1.18, \mathrm{P}=1.03 \times 10^{-3}\right)$ and $\mathrm{rs} 2620381\left(\mathrm{OR}=1.31, \mathrm{P}=2.55 \times 10^{-3}\right)$ remained significant following adjustment for HLA alleles.

\section{Epistasis analysis to identify miRNA interactions}

miRNAs play multiple regulatory roles in both modification of target gene expression and larger regulatory networks. In order to identify epistatic effects between miRNAs and their role in the susceptibility of RA, we applied an epistasis analysis to reveal the interaction between above identified miRNA SNPs. We found 19 SNP-SNP epistatic interactions with $P<7.3 \times 10^{-4}$, indicating significant interactions (Table S3). We found 10 SNP-SNP pairs showed significant strengthened interaction $(O R>1)$ while nine SNP-SNP pairs showed impaired interaction $(\mathrm{OR}<1)$. In addition, enhanced risk epistasis was also identified across HLA alleles (rs4947332 in HLA-DRB1 and rs9275376 in HLA-DQB1) between HLA and miRNAs (HLA-DRB1 and miR3928), and between TNFAIP3 and miR-5695 (Table 4). A significant positive interaction between rs4947332 $(H L A-D R B 1)$ and rs5997893 (miR-3928) with a significantly inflated $\mathrm{OR}=2.83\left(95 \% \mathrm{Cl}: 1.75-4.58, \mathrm{P}=1.36 \times 10^{-5}\right.$, Table 4) for double risk allele carriers, indicating the importance of HLA and non-HLA genetic variation interaction in RA susceptibility.

\section{Cumulative analysis revealed increased risk effect on rheumatoid arthritis}

To estimate the combined effect of the 6 risk alleles (rs1414273T, rs4947332T, rs9268839G, rs9275376T, rs7752903G and rs2620381A) on RA risk, the individual accumulation of risk alleles was treated as an ordinal variable in a logistic regression analysis adjusted by BMI, age and gender. As anticipated, we found the RA risk showed a positive correlation with cumulative number of risk alleles $\left(O R=1.4, P=2.0 \times 10^{-16}, Z=12.54\right.$, $\mathrm{SE}=0.027$, Table 5). In our dataset, the largest subgroup of cases carries five risk alleles (25.6\% of $\mathrm{RA}$ samples) while the largest subgroup of controls carries only 4 risk alleles $(29.4 \%$ of control samples). The OR for RA status for carriers with eight risk alleles (2.9\% of RA population) was 15.38-fold increased over individuals with only 1 risk alleles (10.76\% of normal population). 
To construct regulatory networks between miRNAs and genes, we predicted target genes for the significant miRNAs identified in our study. First, we predicted candidate genes of our significant miRNAs and then mapping these targets to overall immune related genes collected by InnateDB. We found target genes of RAassociated miRNAs were significant enriched in the immune related gene category $\left(P<2.2 \times 10^{-16}\right.$, empirical $\mathrm{P}=2.0 \times 10^{-5}$ and $\mathrm{FC}=1.43$, Figure $3 \mathrm{~A}$, Figure $\mathrm{S} 1 \mathrm{~A}$ ). In addition, we found miR-548ac has 12 target genes overlapping with previous GWAS-identified RA-associated genes including MED1, IL6R, CEP57, CDK6, RAD51B, RUNX1, RTKN2, RAG1, FADS1, RASGRP1, ETS1 and COG6. miR-4308 showed seven candidate genes including RTKN2, TRAF6, RAD51B, PTPN2, PLD4, TNFRSF14 and SYNGR1. miR-3135b showed 4 target genes including GRHL2, CD28, PPIL4 and RASGRP1. Although a hypergeometric test showed miRNA targets significantly enriched in GWAS-identified RA candidate genes $\left(P=7.66 \times 10^{-3}, F C=1.59\right.$, Figure 3B, Table S4), permutation based analysis showed a non-significant enrichment (empirical $P=0.23$ ) indicating an unstable enrichment (Figure S1B). Finally, we also observed miRNA targets also enriched in FDA approved drug targets $\left(P<2.2 \times 10^{-16}\right.$, empirical $\left.P=0.014, F C=1.82\right)$ indicating miRNA as the key regulatory network hubs might be promising drug targets for autoimmune diseases (Figure 3C, Figure S1C).

\section{Discussion}

In this study, 225 East-Asian common SNPs located in human microRNA seed regions were genotyped and association and epistasis analyses were conducted to investigate the association between miRNA and seropositive RA in the Han Chinese population (Shanghai, China). To evaluate the potential association of these East-Asian-specific common miRNA SNPs with RA susceptibility, a case/control study was conducted involving 1,607 seropositive RA patients compared to 1,580 matched controls. The study identified 6 novel RAassociated miRNA SNPs (rs1414273 in miR-548ac, rs2620381 in miR-627, rs4285314 in miR-3135b, rs28477407 in miR-4308; rs5997893 in miR-3928 and rs45596840 in miR-4482) and revealed the interaction between HLA alleles and miRNA SNPs, thereby advancing our understanding of RA pathogenesis.

We found rs45596840 and rs2620381 located in seed regions of miR-4482 and miR-627, respectively, are significantly associated with RA. rs9268839-G (HLA-DRB9) was showed as a risk allele with $\mathrm{OR}=2.47$ in Caucasians population and we found the OR is 1.72 indicating the consistent risk effect and might be required to be validated in African population. According to the miRNA-mRNA binding imputation, rs45596840 (miR4482) and rs2620381 (miR-627) will affect more than 5,894 (2,132 gain and 3,517 loss, see URL) and 4,845 (2,593 gain and 2,252 loss, see URL) mRNA-miRNA bindings respectively. These miRNA target change might change the immune response significantly. In addition, the common regulation network between miR-4707 and miR-627 with CD244, CAMTA1 might be an interesting. rs1414273 is located within the miR-548ac stem-loop sequencing in the first intron of the CD58 gene, which is showed a strong linkage disequilibrium with the Multiple Sclerosis (MS)-associated haplotype. ${ }^{46}$ Hecker M et al. found that SNP rs1414273 might alter Drosha cleavage activity to provoke partial uncoupling of CD58 gene expression and microRNA-548ac production from the shared primary transcript in immune cells and to regulate the inflammatory processes and the balance of protein folding and degradation. ${ }^{47}$ Although $C D 58-C D 2$ interactions recruiting lymphocyte to inflammatory sites to play a crucial role in autoimmune disease. ${ }^{48}{ }^{49}$ Finally, in our previous research, we found DNA methylation of CD4+ cell in rheumatoid arthritis showed abnormal DNA methylation in HLA regions. ${ }^{50}$ In this current study, we found interactions between HLA-miRNA as contributing to RA susceptibility. Overall, the interaction between regulation/epigenetics and genetic variation in RA is a promising field for future research.

There are limitations with our study. Due to the scope of the study, only a limited number of ancestryinformative SNPs were used to control for confounding by population stratification. To validate the RA phenotyping, we utilized 4 East-Asian GWAS RA-associated variants to serve as positive controls for RA samples. We also required all the enrolled individuals were from three-generation Han Chinese family without genetic admixture with other non-Han ethnic individuals to avoid population stratification. This study did not provide functional validation of these miRNAs which is important to show biological validation of the miRNA findings and understand the mechanisms by which these miRNAs are involved in RA susceptibility. Subsequent studies should focus on functional studies of these miRNAs. Overall, we identified 6 miRNA SNPs which are significant associated with the Han Chinese RA population. Two of these SNPs are located in seed 
region of their miRNAs and are expected tol cause target gain/loss, while another three SNPs are located in the loop/pre-miRNA regions which may influence the stability of corresponding miRNAs.

\section{Declarations}

Acknowledgements We thank all participating subjects for their kind cooperation in this study. All methods were performed in accordance with the relevant guidelines and regulations recorded in IRB approved research proposal. All patients were random enrolled and fulfilled the 2010 American College of Rheumatology classification criteria for RA.

Contributors $S G$ and $D H$ contributed to the conception, design and final approval of the submitted version. YJ, YB, JZ, QZ, TJ, YB, RZ, LX, CC, JS, XZ, YS, YQ, JC, XT, PC, QD, YZ, JL, QC, MG, ZL, WQ, YQ, YS, YS, $H N$ developed consent form, recruited individuals, collected blood samples and clinical information collection. SG, YJ contributed to statistical data analysis. JS and DH contributed to the conception, study design, manuscript review and result explanation. The final manuscript was finally completed by SG, YJ, SS and DH. All authors read and approved the final manuscript.

Funding This work was funded by the National Natural Science Funds of China (81774114), Shanghai Chinese Medicine Development Office, Shanghai Chinese and Western Medicine Clinical Pilot Project (ZY(2018-2020)-FWTX-1010), Shanghai Chinese Medicine Development Office, Shanghai Traditional Chinese Medicine Specialty Alliance Project (ZY(2018-2020)-FWTX-4017), National Administration of Traditional Chinese Medicine, Regional Chinese Medicine (Specialist) Diagnosis and Treatment Center Construction Project-Rheumatology.

Competing interests No potential conflicts of interest was disclosed for all the authors.

Patient and public involvement Patients and/or the public were not involved in the design, or conduct, or reporting or dissemination plans of this research.

Patient consent for publication Not required.

Ethical approval On 15 April 2014, the study was reviewed and approved by Guanghua Hospital Research Institute Institutional Review Board (2015-SRA-01, Title: Genetic and Epigenetic Research to Rheumatoid Arthritis).

Data availability statement Summary information are access public and provided in supplementary table for further meta-analysis. In addition, the summary statistic has been deposited in the GitHub page https://github.com/Shicheng-Guo/miRNA-RA. Raw data and materials are available upon the request to the corresponding authors on reasonable collaboration request.

\section{URL:}

dbSNP153: https://ftp.ncbi.nlm.nih.gov/snp/latest_release/VCF/

miRBase: ftp://mirbase.org/pub/mirbase/CURRENTT/README

miRNASNP: http://bioinfo.life.hust.edu.cn/miRNASNP2/index.php

miRDB: http://www.mirdb.org/ontology.html

InnateDB, 4,723 immune genes: https://www.innatedb.com/

rs2273626-target: http://bioinfo.life.hust.edu.cn/miRNASNP\#!/snp?snp_id=rs2273626\&location=Seed\&one=1 rs45596840-target:http://bioinfo.life.hust.edu.cn/miRNASNP\#!/snp?snp_id=rs45596840\&location=Seed\&one=1 rs2620381-targets: http://bioinfo.life.hust.edu.cn/miRNASNP\#!/snp?snp_id=rs2620381\&location=Seed\&one=1

\section{Reference}

1 Stahl EA, Wegmann D, Trynka G, et al. Bayesian inference analyses of the polygenic architecture of rheumatoid arthritis. Nat Genet 2012;44:483-9.

2 van der Woude D, Alemayehu WG, Verduijn W, et al. Gene-environment interaction influences the reactivity of autoantibodies to citrullinated antigens in rheumatoid arthritis. Nat Genet 2010;42:814-6.

3 Cavalli G, Heard E. Advances in epigenetics link genetics to the environment and disease. Nature 2019;571:489-99. 
4 Eyre S, Bowes J, Diogo D, et al. High-density genetic mapping identifies new susceptibility loci for rheumatoid arthritis. Nat Genet 2012;44:1336-40.

5 Westra HJ, Martinez-Bonet M, Onengut-Gumuscu S, et al.Fine-mapping and functional studies highlight potential causal variants for rheumatoid arthritis and type 1 diabetes. Nat Genet 2018;50:1366-74.

6 Raychaudhuri S, Sandor C, Stahl EA, et al. Five amino acids in three HLA proteins explain most of the association between MHC and seropositive rheumatoid arthritis. Nat Genet 2012;44:291-6.

7 Okada Y, Terao C, Ikari K, et al. Meta-analysis identifies nine new loci associated with rheumatoid arthritis in the Japanese population. Nat Genet 2012;44:511-6.

8 Stahl EA, Raychaudhuri S, Remmers EF, et al. Genome-wide association study meta-analysis identifies seven new rheumatoid arthritis risk loci. Nat Genet 2010;42:508-14.

$9 \mathrm{Li} \mathrm{M}$, Santpere G, Imamura Kawasawa Y, et al. Integrative functional genomic analysis of human brain development and neuropsychiatric risks. Science 2018;362.

10 Maurano MT, Humbert R, Rynes E, et al. Systematic localization of common disease-associated variation in regulatory DNA. Science 2012;337:1190-5.

11 Zhu X, Wu L, Mo X, et al. Identification of PBMC-expressed miRNAs for rheumatoid arthritis. Epigenetics 2020;15:386-97.

12 Lai NS, Yu HC, Tung CH, et al. The role of aberrant expression of T cell miRNAs affected by TNF-alpha in the immunopathogenesis of rheumatoid arthritis. Arthritis Res Ther 2017;19:261.

13 Khalifa O, Pers YM, Ferreira R, et al. X-Linked miRNAs Associated with Gender Differences in Rheumatoid Arthritis. Int J Mol Sci 2016;17:1852.

14 Karami J, Aslani S, Tahmasebi MN, et al. Epigenetics in rheumatoid arthritis; fibroblast-like synoviocytes as an emerging paradigm in the pathogenesis of the disease. Immunol Cell Biol 2020;98:171-86.

15 Moran-Moguel MC, Petarra-Del Rio S, Mayorquin-Galvan EE, et al. Rheumatoid Arthritis and miRNAs: A Critical Review through a Functional View. J Immunol Res 2018;2018:2474529.

16 Yang XK, Li P, Zhang C, et al. Association between IRAK1 rs3027898 and miRNA-499 rs3746444 polymorphisms and rheumatoid arthritis : A case control study and meta-analysis. Z Rheumatol 2017;76:62229.

$17 \mathrm{Fu}$ L, Jin L, Yan L, et al. Comprehensive review of genetic association studies and meta-analysis on miRNA polymorphisms and rheumatoid arthritis and systemic lupus erythematosus susceptibility. Hum Immunol 2016;77:1-6.

$18 \mathrm{Li} \mathrm{K}$, Tie H, Hu N, et al. Association of two polymorphisms rs2910164 in miRNA-146a and rs3746444 in miRNA-499 with rheumatoid arthritis: a meta-analysis. Hum Immunol 2014;75:602-8.

19 Yang B, Chen J, Li Y, et al. Association of polymorphisms in pre-miRNA with inflammatory biomarkers in rheumatoid arthritis in the Chinese Han population. Hum Immunol 2012;73:101-6.

20 Hashemi M, Eskandari-Nasab E, Zakeri Z, et al. Association of pre-miRNA-146a rs2910164 and premiRNA499 rs3746444 polymorphisms and susceptibility to rheumatoid arthritis. Mol Med Rep 2013;7:287-91.

21 Shaker OG, El Boghdady NA, El Sayed AE. Association of MiRNA-146a, MiRNA-499, IRAK1 and PADI4 Polymorphisms with Rheumatoid Arthritis in Egyptian Population. Cell Physiol Biochem 2018;46:2239-49.

22 Jiang L, Yin J, Ye L, et al. Novel risk loci for rheumatoid arthritis in Han Chinese and congruence with risk variants in Europeans. Arthritis Rheumatol 2014;66:1121-32.

23 Kozomara A, Birgaoanu M, Griffiths-Jones S. miRBase: from microRNA sequences to function. Nucleic Acids Res 2019;47:D155-D62.

24 Jiang $X Y$, Zhang $Q$, Chen $P$, et al. CYP7A1 polymorphism influences the LDL cholesterol-lowering response to atorvastatin. Int J Clin Pharm 2012;37:719-23.

25 Huang L, Li Y, Guo S, et al. Different hereditary contribution of the CFH gene between polypoidal choroidal vasculopathy and age-related macular degeneration in Chinese Han people. Invest Ophthalmol Vis Sci 2014;55:2534-8.

26 Shen F, Chen J, Guo S, et al. Genetic variants in miR-196a2 and miR-499 are associated with susceptibility to esophageal squamous cell carcinoma in Chinese Han population. Tumour Biol 2016;37:4777-84.

27 Lek M, Karczewski KJ, Minikel EV, et al. Analysis of protein-coding genetic variation in 60,706 humans. Nature 2016;536:285-91.

28 GenomeAsia KC. The GenomeAsia 100K Project enables genetic discoveries across Asia. Nature 2019;576:106-11.

29 Genomes Project C, Auton A, Brooks LD, et al. A global reference for human genetic variation. Nature 2015;526:68-74. 
30 Belsare S, Levy-Sakin M, Mostovoy Y, et al. Evaluating the quality of the 1000 genomes project data. BMC Genomics 2019;20:620.

31 Okada Y, Wu D, Trynka G, et al. Genetics of rheumatoid arthritis contributes to biology and drug discovery. Nature 2014;506:376-81.

32 Laufer VA, Tiwari HK, Reynolds RJ, et al. Genetic influences on susceptibility to rheumatoid arthritis in African-Americans. Hum Mol Genet 2019;28:858-74.

33 Regueiro C, Gonzalez A. Questions on 'Sequencing of the MHC region defines HLA-DQA1 as the major genetic risk for seropositive rheumatoid arthritis in Han Chinese population' by Guo et al. Ann Rheum Dis 2020 34 Guo J, Zhang T, Cao H, et al. Sequencing of the MHC region defines HLA-DQA1 as the major genetic risk for seropositive rheumatoid arthritis in Han Chinese population. Ann Rheum Dis 2019;78:773-80.

35 Song X, Guo S, Chen Y, et al. Association between HLA-DQA1 gene copy number polymorphisms and susceptibility to rheumatoid arthritis in Chinese Han population. J Genet 2014;93:215-8.

36 Castro F, Acevedo E, Ciusani E, et al. Tumour necrosis factor microsatellites and HLA-DRB1* HLA-DQA1*, and HLA-DQB1* alleles in Peruvian patients with rheumatoid arthritis. Ann Rheum Dis 2001;60:791-5.

37 Qin P, Li Z, Jin W, et al. A panel of ancestry informative markers to estimate and correct potential effects of population stratification in Han Chinese. Eur J Hum Genet 2014;22:248-53.

38 Chen Y, Wang X. miRDB: an online database for prediction of functional microRNA targets. Nucleic Acids Res 2020;48:D127-D31.

39 Breuer K, Foroushani AK, Laird MR, et al. InnateDB: systems biology of innate immunity and beyond-recent updates and continuing curation. Nucleic Acids Res 2013;41:D1228-33.

40 Banerjee S, Zeng L, Schunkert H, et al. Bayesian multiple logistic regression for case-control GWAS. PLoS Genet 2018;14:e1007856.

41 Landa I, Ruiz-Llorente S, Montero-Conde C, et al. The variant rs1867277 in FOXE1 gene confers thyroid cancer susceptibility through the recruitment of USF1/USF2 transcription factors. PLoS Genet 2009;5:e1000637.

42 Boulesteix AL, Strobl C, Weidinger S, et al. Multiple testing for SNP-SNP interactions. Stat Appl Genet Mol Biol 2007;6.

43 Purcell S, Neale B, Todd-Brown K, et al. PLINK: a tool set for whole-genome association and populationbased linkage analyses. Am J Hum Genet 2007;81:559-75.

44 Ghosh D. Wavelet-based Benjamini-Hochberg procedures for multiple testing under dependence. Math Biosci Eng 2019;17:56-72.

45 Gong J, Liu C, Liu W, et al. An update of miRNASNP database for better SNP selection by GWAS data, miRNA expression and online tools. Database (Oxford) 2015;2015.

46 Hecker M, Fitzner B, Blaschke J, et al. Susceptibility variants in the CD58 gene locus point to a role of microRNA-548ac in the pathogenesis of multiple sclerosis. Mutat Res Rev Mutat Res 2015;763:161-7.

47 Hecker M, Boxberger N, Illner N, et al. A genetic variant associated with multiple sclerosis inversely affects the expression of CD58 and microRNA-548ac from the same gene. PLoS Genet 2019;15:e1007961.

48 Sable R, Durek T, Taneja V, et al. Constrained Cyclic Peptides as Immunomodulatory Inhibitors of the CD2:CD58 Protein-Protein Interaction. ACS Chem Biol 2016;11:2366-74.

49 Hoffmann JC, Bayer B, Zeidler $\mathrm{H}$. Characterization of a soluble form of CD58 in synovial fluid of patients with rheumatoid arthritis (RA). Clin Exp Immunol 1996;104:460-6.

50 Guo S, Zhu Q, Jiang T, et al. Genome-wide DNA methylation patterns in CD4+ T cells from Chinese Han patients with rheumatoid arthritis. Mod Rheumatol 2017;27:441-47. 
Figure 1. Flowchart for SNP and sample selection as well as population ancestry of GHRA cohort. (A) human microRNAs were downloaded from miRBase (Release 22.1). We mapped all the dbSNP153 SNPs into human Asian 100K data with MAF>1\%. 4 positive control (HLA-DRB1, HLA-DRB9, HLA-DQB1 and TNFAIP3) and 4 ancestry markers (South and North Han Chinese) were added for the quality control. (B) PCA analysis to show the population ancestry of Guanghua Rheumatoid Arthritis cohort (GHRA) with 1000 Genome dataset. Symbols are as same as Super Population Code (SPC) from 1000 genome project. AFR, African; AMR, Ad Mixed American; EAS, East Asian; EUR, European; SAS, South Asian.

Figure 2. Genomic position and functional annotation to the significant SNPs in miRNAs. rs 1414273 $(\mathrm{C} / \mathrm{T})$ in miR-548ac; rs2620381(A/C) in miR-627-5p which cause 2,056 target gain and 1,440 target loss. rs4285314(G/A) in miR-3135b; rs28477407(C/T) in miR-4308; rs5997893 (A/G) in miR-3928-5p and rs45596840 (G/A) in miR-4482-5p which will cause 1,677 target gain and 1,456 target loss. Target gain or loss were imputed by miRNA-SNP database ${ }^{45}$ (see method).

Figure 3. miRNA target analysis and target distribution in GWAS significant genes, immune genes and FDA drug target genes. (A) Network connection between the top 6 miRNA predicted regulatory targets and previous GWAS identified 123 RA associated genes. (B) Network connection between the top 6 miRNA predicted regulatory targets and 4,723 InnateDB collected immune genes. (C) Network connection between the top 6 miRNA predicted regulatory targets and 672 FDA approved drug target genes. miRNA was labelled as diamond filled with green color and gene were as circle filled with yellow color.

Table 1. Summary of risk alleles, 1000 Genome frequencies and SNPs associated with rheumatoid arthritis. *FDR correction were not showed in the table while only $\mathrm{P}<2.55 \times 10^{-3}$ is significant in the table with FDR correction. EAS, EUR and AFR represent allele frequency of A1 in East Asian, European and African population based on 1000 Genome dataset. All the OR and P-value are referring to A1 while taking A2 as reference. Bayesian logistic regression model adjusted for covariates including gender, age and BMI, were applied as the statistical approach.

Table 2. Association statistics for rheumatoid arthritis susceptibility and genetic variants. \& indicating $\mathrm{P}$-values from genotype-based chi-square or fisher exact test (when $\mathrm{N}<=5$ ) models. All the OR and $\mathrm{P}$-value are calculated compared with homogeneous protective allele (reference row as the table). Chi-square or Fisher exact test were applied as the statistical approach so that ORs are comparative between different models.

Table 3. meta-analysis between our study and previous East-Asian population-based RA GWAS study.

Table 4. Epistasis analysis to identify SNP-SNP interaction in rheumatoid arthritis susceptibility. Five significant epistasis effect were selected to show the increased or decreased risk effect. rs4947332 located in C2 upstream (HLA-DRB1); rs9275376 located in HLA-DQB1 upstream; rs5997893 located in miR-3928; rs7752903 located in downstream of TNFAIP3 and rs2967897 located in miR-5695.

Table 5. Cumulative risk effect of rheumatoid arthritis increased as more risk alleles carried by the patients. *individuals carrying more than 9 risk alleles were not found in our dataset. rs1414273 (T), rs4947332 (T), rs9268839 (G), rs9275376 (T), rs7752903 (G) and rs2620381 (A) were included in the analysis. Risk alleles were showed in the parentheses followed the SNPs.

\section{Supplementary Matreial:}

Figure S1.Re-sampling technique based enrichment analysis to miRNA regulatory targets. *A random sampling technique was applied to assess the null distribution of $p$-values ( $n=50,000$ iterations).37,875 total genes in the genome (GENCODE v32) were assumed for the calculations. Enrichment was measured with fold-change (FC) and p-values were calculated by summing the frequency of more extreme values in the null distribution of $p$-values.

Table S1. Demographic information for the GHRA cohort 
Table S2. Cochran-Armitage Trend test-based association analyses.

Table S3. Epistasis analysis to identify SNP-SNP interaction in rheumatoid arthritis susceptibility

Table S4. miRDB based miRNA target prediction for the 6 significant RA-miRNA.
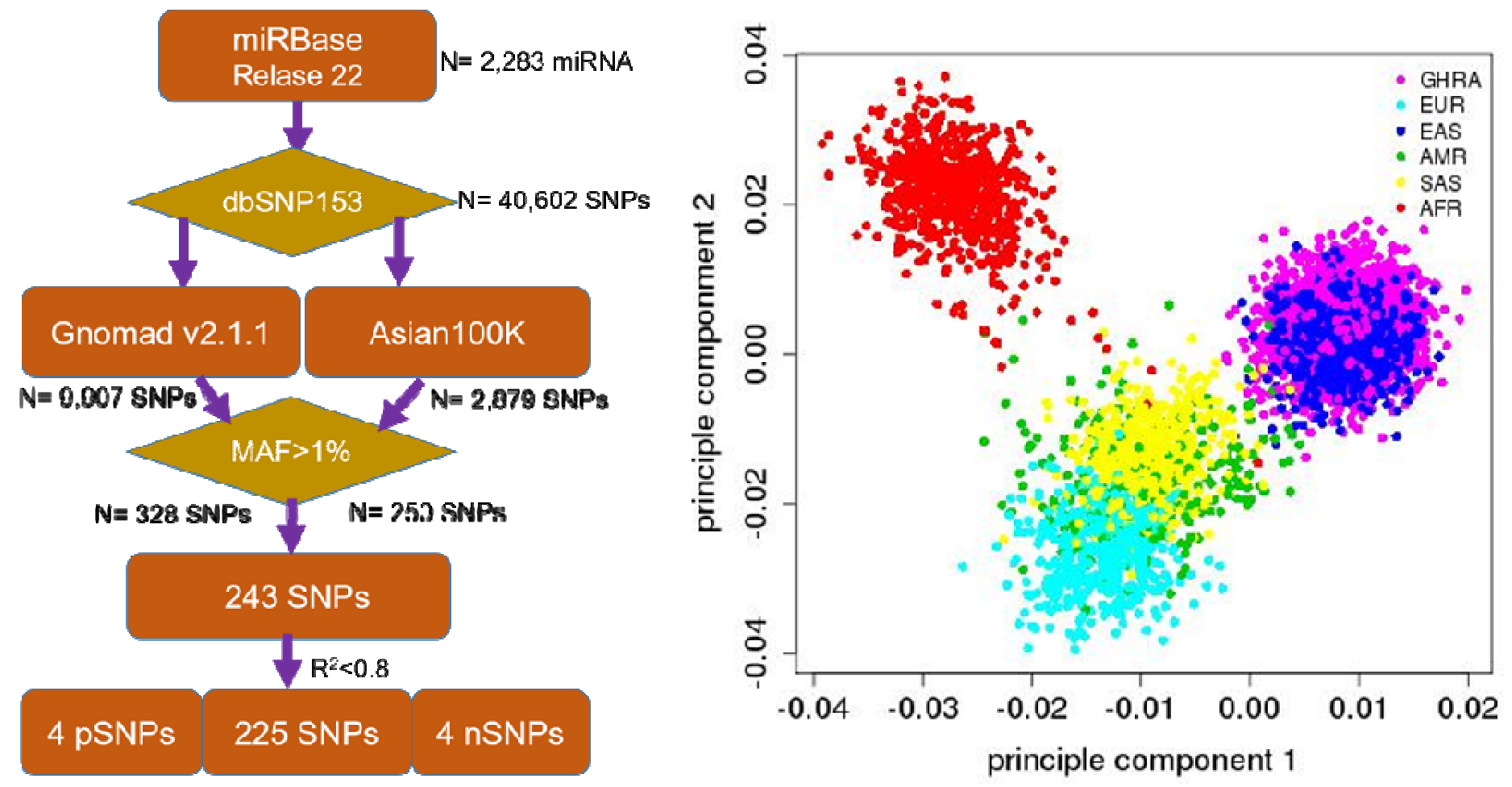

Figure 1. Flowchart for SNP and sample selection as well as population ancestry of GHRA cohort.

(A) human microRNAs were downloaded from miRBase (Release 22.1). We mapped all the dbSNP153 SNPs into human microRNA genomic regions and 40,602 SNPs were received. We filter out non-East common variants with Gnomad and Asian 100K data with MAF>1\%. 4 positive control (HLA-DRB1, HLA-DRB9, HLA-DQB1 and TNFAIP3) and 4 ancestry markers (South and North Han Chinese) were added for the quality control (B) PCA analysis to show the population ancestry of Guanghua Rheumatoid Arthritis cohort (GHRA) with 1000 Genome dataset. Symbols are as same as Super Population Code (SPC) from 1000 genome project. AFR, African; AMR, Ad Mixed American; EAS, East Asian; EUR, European; SAS, South Asian. 


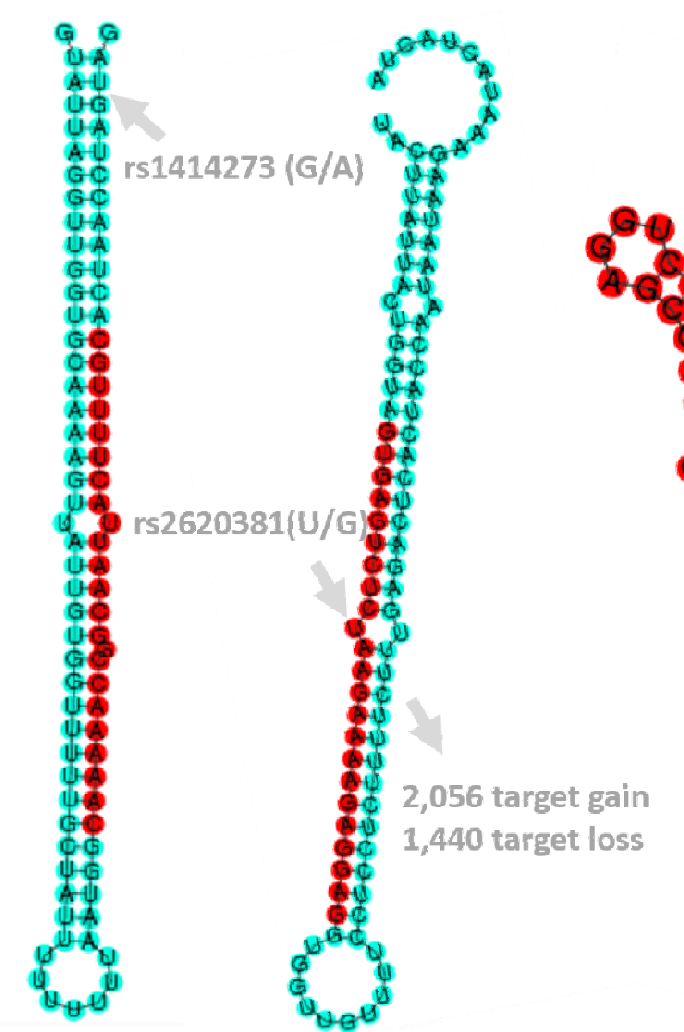

miR-548ac

miR-627

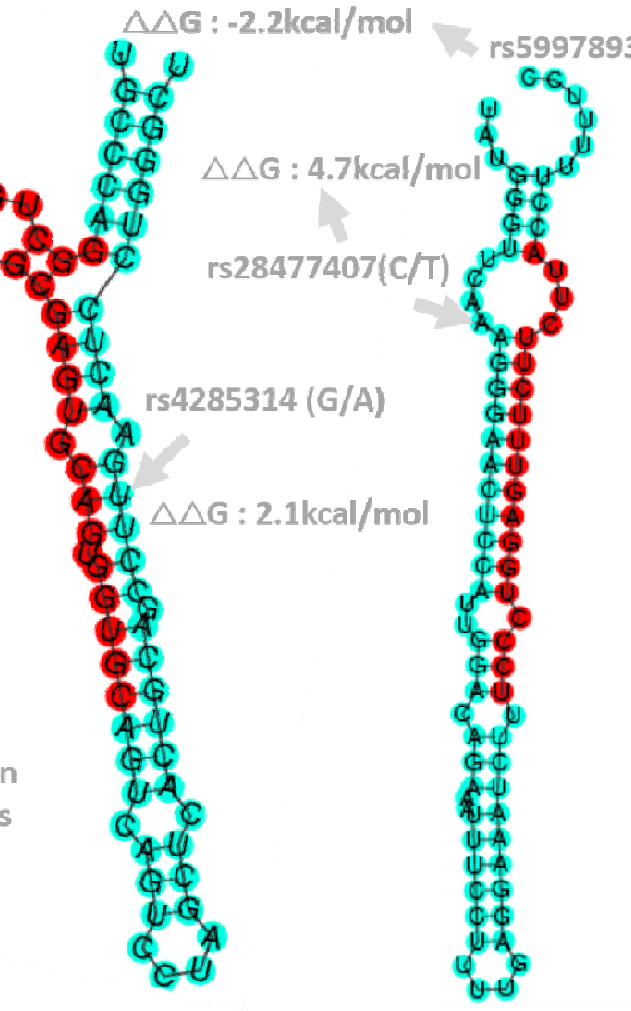

miR-3135b

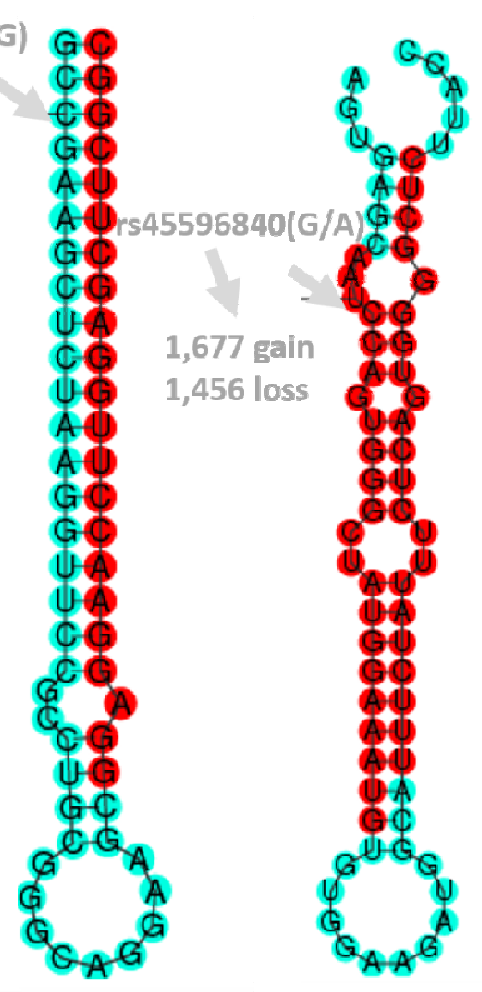

miR-3928

miR-4482

Figure 2. Genomic position and functional annotation to the significant SNPs in miRNAs

rs1414273 (C/T) in miR-548ac; rs2620381(A/C) in miR-627-5p which cause 2056 target gain and 1440 target loss. rs4285314(G/A) in miR-3135b; rs28477407(C/T) in miR-4308; rs5997893 (A/G) in miR-3928-5p and rs45596840 (G/A) in miR-4482-5p which will cause 1677 target gain and 1456 target loss. Target gain or loss were imputed by miRNA-SNP database ${ }^{45}$ (see method). 
A
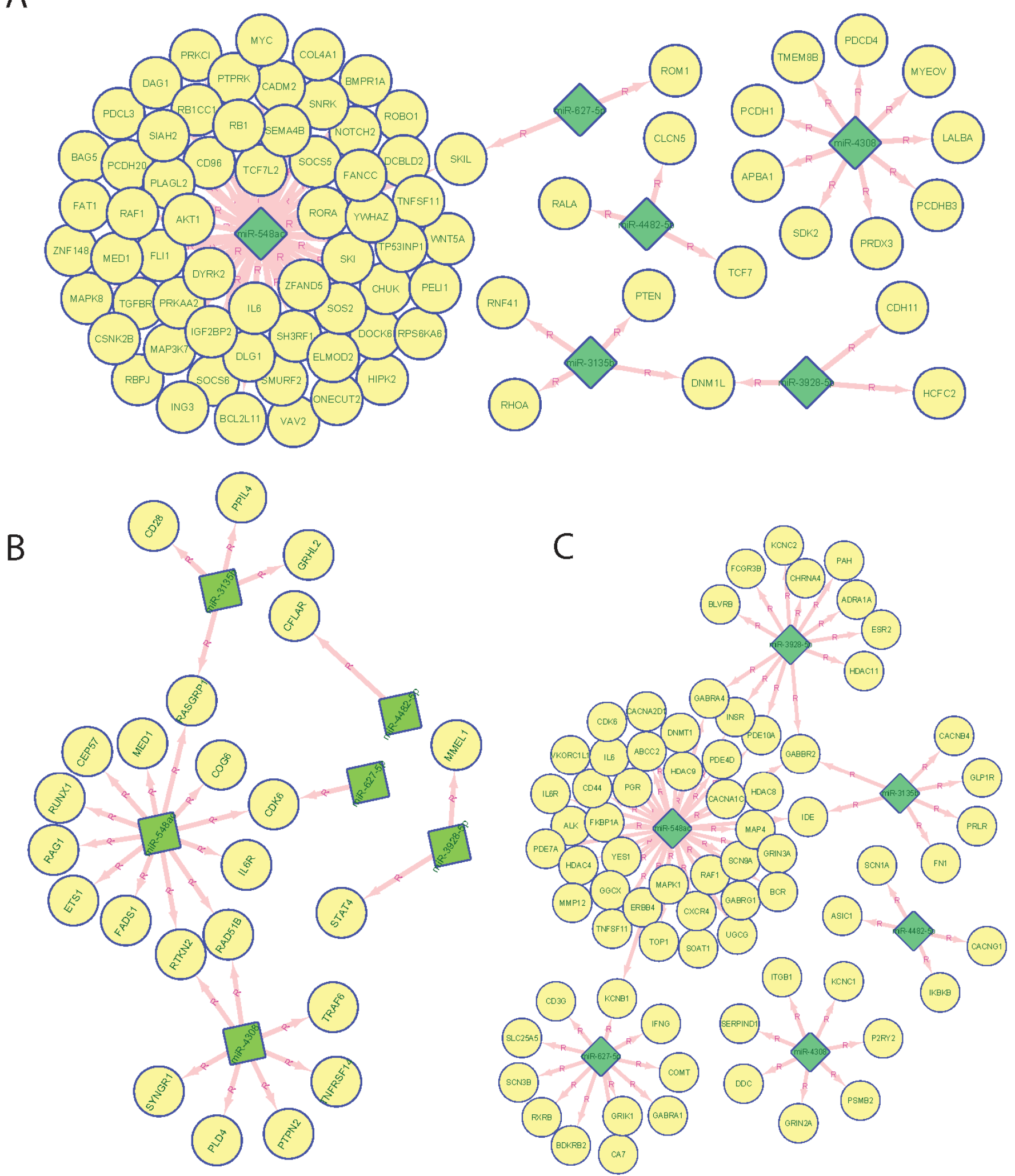

Figure 3. miRNA target analysis and target distribution in GWAS significant genes, immune genes and FDA drug target genes. (A). Network connection between the top 6 miRNA predicted regulatory targets and previous GWAS identified 123 RA associated genes. (B). Network connection between the top 6 miRNA predicted regulatory targets and 4,723 InnateDB collected immune genes. (C) Network connection between the top 6 miRNA predicted regulatory targets and 672 FDA approved drug target genes. miRNA was labelled as diamond filled with green color and gene were as circle filled with yellow color. 
Table 1. Summary of risk alleles, 1000 Genome frequencies and SNPs associated with rheumatoid arthritis

\begin{tabular}{|c|c|c|c|c|c|c|c|c|c|}
\hline $\mathrm{CHR}$ & SNP & $\mathrm{A} 1 / \mathrm{A} 2$ & RA (no.) & Normal (no.) & OR & $\mathrm{P}$ & EAS & EUR & AFR \\
\hline 6p21.32 & rs9268839 & $\mathrm{A} / \mathrm{G}$ & $42.4 \%(1364)$ & $56.0 \%(1768)$ & $0.58(0.53-0.64)$ & $3.95 \times 10^{-27^{*}}$ & 0.354 & 0.451 & 0.236 \\
\hline 6p21.32 & rs9275376 & $\mathrm{T} / \mathrm{G}$ & $25.0 \%(804)$ & $15.7 \%(496)$ & $1.79(1.58-2.03)$ & $2.65 \times 10^{-20^{*}}$ & 0.192 & 0.299 & 0.285 \\
\hline $6 q 23.3$ & rs7752903 & $\mathrm{G} / \mathrm{T}$ & $6.4 \%(205)$ & $4.3 \%(136)$ & $1.51(1.21-1.89)$ & $2.33 \times 10^{-4^{*}}$ & 0.049 & 0.019 & 0.056 \\
\hline $6 p 21.33$ & rs4947332 & $\mathrm{T} / \mathrm{C}$ & $2.2 \%(72)$ & $1.1 \%(34)$ & $2.11(1.40-3.18)$ & $2.78 \times 10^{-4^{*}}$ & 0.012 & 0.029 & 0.094 \\
\hline $1 \mathrm{p} 13.1$ & rs1414273 & $\mathrm{C} / \mathrm{T}$ & $40.5 \%(1303)$ & $44.7 \%(1412)$ & $0.84(0.76-0.93)$ & $8.26 \times 10^{-4^{*}}$ & 0.585 & 0.140 & 0.584 \\
\hline $15 q 15.1$ & rs2620381 & $\mathrm{C} / \mathrm{A}$ & $7.9 \%(254)$ & $10.1 \%(318)$ & $0.77(0.65-0.91)$ & $2.55 \times 10^{-3^{*}}$ & 0.086 & 0.004 & 0.130 \\
\hline $14 q 32.31$ & rs75330474 & $\mathrm{T} / \mathrm{C}$ & $3.8 \%(121)$ & $5.0 \%(159)$ & $0.74(0.58-0.94)$ & 0.01360 & 0.065 & 0.003 & 0.064 \\
\hline $18 p 11.21$ & rs370878033 & $\mathrm{G} / \mathrm{A}$ & $1.7 \%(54)$ & $2.6 \%(81)$ & $0.65(0.46-0.92)$ & 0.01435 & 0.032 & 0.001 & 0.001 \\
\hline $7 q 22.1$ & rs3823658 & $\mathrm{A} / \mathrm{G}$ & $13.4 \%(432)$ & $11.5 \%(363)$ & $1.20(1.03-1.39)$ & 0.01825 & 0.138 & 0.138 & 0.006 \\
\hline $1 p 32.3$ & rs74085143 & $\mathrm{A} / \mathrm{G}$ & $4.0 \%(130)$ & $5.3 \%(166)$ & $0.76(0.60-0.96)$ & 0.02190 & 0.048 & 0.022 & 0.331 \\
\hline $6 q 23.2$ & rs7740161 & $\mathrm{T} / \mathrm{A}$ & $14.3 \%(459)$ & $16.3 \%(515)$ & $0.86(0.75-0.98)$ & 0.02530 & 0.102 & 0.347 & 0.614 \\
\hline $3 p 21.1$ & rs4687672 & $\mathrm{A} / \mathrm{G}$ & $33.7 \%(1082)$ & $31.1 \%(983)$ & $1.12(1.01-1.25)$ & 0.02914 & 0.317 & 0.264 & 0.202 \\
\hline $22 q 12.2$ & rs5997893 & $\mathrm{A} / \mathrm{G}$ & $46.9 \%(1509)$ & $49.5 \%(1565)$ & $0.90(0.82-1.00)$ & 0.03972 & 0.498 & 0.667 & 0.918 \\
\hline 19p13.2 & rs2967897 & $\mathrm{T} / \mathrm{C}$ & $16.8 \%(541)$ & $15.0 \%(473)$ & $1.15(1.00-1.31)$ & 0.04189 & 0.849 & 0.686 & 0.614 \\
\hline $15 q 25.3$ & rs76468441 & $\mathrm{T} / \mathrm{C}$ & $3.2 \%(104)$ & $2.4 \%(76)$ & $1.36(1.00-1.83)$ & 0.04530 & 0.042 & 0.054 & 0.011 \\
\hline
\end{tabular}

${ }^{*}$ FDR correction were not showed in the table while only $\mathrm{P}<2.55 \times 10^{-3}$ is significant in the table with FDR correction. EAS, EUR and AFR represent allele frequency of A1 in East Asian, European and African population based on 1000 Genome dataset. All the $\mathrm{OR}$ and $\mathrm{P}$-value are referring to $\mathrm{A} 1$ while taking $\mathrm{A} 2$ as reference. Bayesian logistic regression model adjusted for covariates including gender, age and BMI, were applied as the statistical approach. 
Table 2. Association statistics for rheumatoid arthritis susceptibility and genetic variants

\begin{tabular}{|c|c|c|c|c|}
\hline Genotypes, risk allele & $\mathrm{RA}$ & Normal & OR $(95 \% \mathrm{Cl})$ & P-value \\
\hline \multicolumn{5}{|c|}{ rs9268839, located at 6p21.32, HLA-DRB9 } \\
\hline AA & $262(16.30 \%)$ & $494(31.27 \%)$ & 1.00 (reference) & \multirow[t]{3}{*}{$1.46 \times 10^{-27 \&}$} \\
\hline GA & $840(52.27 \%)$ & $780(49.37 \%)$ & $2.03(1.69-2.44)$ & \\
\hline GG & $505(31.43 \%)$ & $306(19.37 \%)$ & $3.11(2.52-3.85)$ & \\
\hline$G G$ vs $G A+A A$ & $505(31.43 \%)$ & $306(19.37 \%)$ & $1.91(1.62-2.25)$ & $4.77 \times 10^{-15}$ \\
\hline$G G+G A$ vs $A A$ & $1345(83.70 \%)$ & $1086(68.73 \%)$ & $2.34(1.96-2.78)$ & $2.01 \times 10^{-23}$ \\
\hline G allele & $1850(57.56 \%)$ & $1392(44.05 \%)$ & $1.72(1.56-1.9)$ & $3.86 \times 10^{-27}$ \\
\hline \multicolumn{5}{|c|}{ rs9275376, located at 6p21.32, $H L A-D Q B 1$} \\
\hline GG & $887(55.20 \%)$ & $1128(71.39 \%)$ & 1.00 (reference) & \multirow[t]{3}{*}{$1.26 \times 10^{-20 \&}$} \\
\hline TG & $636(39.58 \%)$ & $408(25.82 \%)$ & $1.98(1.70-2.32)$ & \\
\hline TT & $84(5.23 \%)$ & $44(2.78 \%)$ & $2.43(1.65-3.62)$ & \\
\hline TT vs TG+GG & $84(5.23 \%)$ & $44(2.78 \%)$ & $1.93(1.31-2.86)$ & $5.58 \times 10^{-4}$ \\
\hline TT+TG vs GG & $720(44.80 \%)$ & $452(28.61 \%)$ & $2.03(1.74-2.35)$ & $2.05 \times 10^{-21}$ \\
\hline T allele & $804(25.02 \%)$ & $496(15.70 \%)$ & $1.79(1.58-2.03)$ & $2.01 \times 10^{-20}$ \\
\hline \multicolumn{5}{|c|}{ rs7752903, located at 6q23.3, TNFAIP3 } \\
\hline TT & $1408(87.62 \%)$ & $1445(91.46 \%)$ & 1.00 (reference) & \multirow[t]{3}{*}{$4.19 \times 10^{-4 \&}$} \\
\hline GT & $193(12.01 \%)$ & $134(8.48 \%)$ & $1.48(1.16-1.88)$ & \\
\hline GG & $6(0.37 \%)$ & $1(0.06 \%)$ & $6.16(0.75-283.06)$ & \\
\hline GG vs GT+TT & $6(0.37 \%)$ & $1(0.06 \%)$ & $5.92(0.72-272.02)$ & 0.12460 \\
\hline $\mathrm{GG}+\mathrm{GT}$ vs TT & $199(12.38 \%)$ & $135(8.54 \%)$ & $1.51(1.19-1.92)$ & $4.12 \times 10^{-4}$ \\
\hline G allele & $205(6.38 \%)$ & $136(4.30 \%)$ & $1.51(1.21-1.91)$ & $2.33 \times 10^{-4}$ \\
\hline \multicolumn{5}{|c|}{ rs4947332, located at 6p21.33, Tag-SNPs of $H L A-D R B 1^{*} 04: 07$} \\
\hline $\mathrm{CC}$ & $1537(95.64 \%)$ & $1546(97.85 \%)$ & 1.00 (reference) & \multirow[t]{3}{*}{$5.51 \times 10^{-4 \&}$} \\
\hline TC & $68(4.23 \%)$ & $34(2.15 \%)$ & $2.01(1.31-3.15)$ & \\
\hline TT & $2(0.12 \%)$ & $0(0.00 \%)$ & - & \\
\hline TT vs TC+CC & $2(0.12 \%)$ & $0(0.00 \%)$ & - & 0.49980 \\
\hline TT+TC vs CC & $70(4.36 \%)$ & $34(2.15 \%)$ & $2.07(1.35-3.24)$ & $4.53 \times 10^{-4}$ \\
\hline T allele & $72(2.24 \%)$ & $34(1.08 \%)$ & $2.11(1.38-3.28)$ & $2.67 \times 10^{-4}$ \\
\hline \multicolumn{5}{|c|}{ rs1414273, located at $1 p 13.1$ within miR-548ac } \\
\hline $\mathrm{CC}$ & $284(17.67 \%)$ & $307(19.43 \%)$ & 1.00 (reference) & \multirow[t]{3}{*}{$4.84 \times 10^{-4 \&}$} \\
\hline TC & $735(45.74 \%)$ & $798(50.51 \%)$ & $1.00(0.82-1.21)$ & \\
\hline TT & $588(36.59 \%)$ & $475(30.06 \%)$ & $1.34(1.09-1.65)$ & \\
\hline TT vs TC+CC & $588(36.59 \%)$ & $475(30.06 \%)$ & $1.34(1.15-1.56)$ & $9.35 \times 10^{-5}$ \\
\hline TT+TC vs CC & $1323(82.33 \%)$ & $1273(80.57 \%)$ & $1.12(0.94-1.35)$ & 0.20230 \\
\hline T allele & $1911(59.46 \%)$ & $1748(55.32 \%)$ & $1.18(1.07-1.31)$ & $9.04 \times 10^{-4}$ \\
\hline \multicolumn{5}{|c|}{ rs2620381, located at $15 q 15.1$ within $\operatorname{miR}-627$} \\
\hline $\mathrm{CC}$ & $9(0.56 \%)$ & $20(1.27 \%)$ & 1.00 (reference) & \multirow[t]{3}{*}{$7.27 \times 10^{-3 \&}$} \\
\hline$A C$ & $236(14.69 \%)$ & $278(17.59 \%)$ & $1.89(0.8-4.79)$ & \\
\hline $\mathrm{AA}$ & $1362(84.75 \%)$ & $1282(81.14 \%)$ & $2.36(1.02-5.91)$ & \\
\hline$A A$ vs $A C+C C$ & $1362(84.75 \%)$ & $1282(81.14 \%)$ & $1.29(1.07-1.56)$ & $7.21 \times 10^{-3}$ \\
\hline$A A+A C$ vs $C C$ & $1598(99.44 \%)$ & $1560(98.73 \%)$ & $2.28(0.99-5.7)$ & 0.04035 \\
\hline A allele & $2960(92.10 \%)$ & $2842(89.94 \%)$ & $1.30(1.09-1.56)$ & 0.00285 \\
\hline
\end{tabular}

\& indicating P-values from genotype-based chi-square or fisher exact test (when $\mathrm{N}<=5$ ) models. All the $\mathrm{OR}$ and $\mathrm{P}$-value are calculated compared with homogeneous protective allele (reference row as the table). Chi-square or Fisher exact test were applied as the statistical approach so that ORs are comparative between different models. 
Table 3. meta-analysis between our study and previous East-Asian population-based RA GWAS study

\begin{tabular}{lllllll}
\hline CHR & \multicolumn{1}{c}{ SNP } & BP & A1 & $\mathrm{OR}^{1}$ & $\mathrm{P}^{1}$ & FDR \\
\hline 6 & rs9268839 (HLA-DRB9) & 32428772 & $\mathrm{~A}$ & 0.52 & $4.15 \times 10^{-49}$ & $2.08 \times 10^{-4 /}$ \\
6 & rs9275376 (HLA-DQB1) & 32668633 & $\mathrm{~T}$ & 1.72 & $1.11 \times 10^{-47}$ & $1.04 \times 10^{-45}$ \\
6 & rs7752903 (TNFAIP3) & 138227364 & $\mathrm{~T}$ & 0.73 & $1.94 \times 10^{-22}$ & $1.21 \times 10^{-20}$ \\
6 & rs4285314(miR-3135b) & 32717702 & $\mathrm{~A}$ & 1.33 & $2.35 \times 10^{-15}$ & $1.10 \times 10^{-13}$ \\
14 & rs28477407(miR-4308) & 55344901 & $\mathrm{~T}$ & 0.89 & $9.19 \times 10^{-07}$ & $3.44 \times 10^{-5}$ \\
22 & rs5997893(miR-3928) & 31556103 & $\mathrm{~A}$ & 0.93 & $1.92 \times 10^{-04}$ & $5.97 \times 10^{-3}$ \\
10 & rs45596840 (miR-4482) & 106028154 & $\mathrm{~A}$ & 0.89 & $2.49 \times 10^{-04}$ & $6.65 \times 10^{-3}$ \\
6 & rs4947332(HLA-DRB1) & 31834197 & $\mathrm{~T}$ & 1.41 & $9.50 \times 10^{-04}$ & $2.22 \times 10^{-2}$ \\
\hline
\end{tabular}

Table 4. Epistasis analysis to identify SNP-SNP interaction in rheumatoid arthritis susceptibility

\begin{tabular}{lllllll}
\hline Epistasis & \multicolumn{2}{c}{ SNP Genotypes } & RA & Control & OR & P \\
\hline \multirow{2}{*}{ rs4947332\& rs9275376 } & rs4947332 & rs9275376 & & & & \\
& CC & GG & 885 & 1119 & 1.00 (reference) & - \\
& CT+TT & GG & 2 & 9 & $0.38(0.10-1.38)$ & 0.164 \\
& CC & GT+TT & 652 & 427 & $1.93(1.66-2.24)$ & $6.59 \times 10^{-18}$ \\
& CT+TT & GT+TT & 68 & 25 & $3.35(2.12-5.31)$ & $6.70 \times 10^{-8}$ \\
& & & & & & - \\
rs4947332\& rs5997893 & rs4947332 & rs5997893 & & & & \\
& CC & GG & 330 & 392 & 1.00 (reference) & - \\
& CT+TT & GG & 9 & 9 & $1.19(0.49-2.89)$ & 0.821 \\
& CC & GT+TT & 1207 & 1154 & $1.24(1.05-1.47)$ & 0.012 \\
& CT+TT & GT+TT & 61 & 25 & $2.83(1.75-4.58)$ & $1.36 \times 10^{-5}$ \\
& & & & & & \\
rs7752903\& rs2967897 & rs7752903 & rs2967897 & & & & - \\
& TT & CC & 953 & 1050 & 1.00 (reference) & - \\
& TG+GG & CC & 148 & 93 & $1.75(1.33-2.29)$ & $5.76 \times 10^{-5}$ \\
& TT & CT+TT & 455 & 395 & $1.27(1.08-1.49)$ & 0.00370 \\
& TG+GG & CT+TT & 51 & 42 & $1.33(0.88-2.01)$ & 0.20705 \\
\hline
\end{tabular}

Five significant epistasis effect were selected to show the increased or decreased risk effect. rs4947332 located in C2 upstream (HLA-DRB1); rs9275376 located in HLA-DQB1 upstream; rs5997893 located in miR-3928; rs7752903 located in downstream of TNFAIP3 and rs2967897 located in miR-5695.

Table 5. Cumulative risk effect of rheumatoid arthritis increased as more risk alleles carried by the patients

\begin{tabular}{lllll}
\hline No. of risk alleles* & RA & Normal & OR $(95 \%$ Cl) & P \\
\hline 1 & 4 & 17 & 1.00 (Reference) & - \\
2 & 65 & 133 & $1.77(0.63-4.99)$ & 0.348209 \\
3 & 206 & 340 & $2.19(0.80-5.97)$ & 0.129698 \\
4 & 388 & 466 & $3.00(1.10-8.15)$ & 0.031822 \\
5 & 411 & 359 & $4.12(1.51-11.21)$ & 0.002819 \\
6 & 344 & 185 & $6.68(2.44-18.27)$ & $6.30 \times 10^{-05}$ \\
7 & 138 & 67 & $7.36(2.62-20.66)$ & $4.26 \times 10^{-05}$ \\
8 & 46 & 10 & $15.38(4.69-50.49)$ & $1.00 \times 10^{-06}$ \\
9 & 5 & 3 & $5.40(1.08-26.93)$ & 0.049413 \\
\hline
\end{tabular}

${ }^{*}$ individuals carrying 0 or more than 9 risk alleles was not found in our dataset. rs1414273 (T), rs4947332 (T), rs9268839 $(\mathrm{G})$, rs9275376 (T), rs7752903 (G) and rs2620381 (A) were included in the analysis. Risk alleles were showed in the parentheses followed the SNPs. 